\title{
Thema \\ Empirische Bildungsforschung im deutschsprachigen Raum. Rückblick und Ausblick
}

\author{
Katharina Maag Merki, Universität Zürich
}

Der Aufschwung der empirischen Bildungsforschung im deutschsprachigen Raum in den letzten zwanzig Jahren steht in einem Zusammenhang mit den Ergebnissen der international vergleichenden Leistungsstudien wie PISA und TIMSS, der Implementation neuer Steuerungsformen sowie mit umfangreichen strukturellen Massnahmen. In diesem Artikel erfolgt zunächst eine Auseinandersetzung mit der "empirischen Bildungsforschung» als Forschungsfeld. In einem zweiten Schritt werden aktuelle Forschungslücken beschrieben. Forschungsbedarf kann in theoretischer und methodischer Perspektive und bezüglich der Klärung der Fragen nach den Bedingungsfaktoren und Effekten von Bildungsprozessen identifiziert werden. Des Weiteren wird für den Aufbau einer Forschungsinfrastruktur für Bildungsforschung plädiert.

\section{Einleitung}

Der Aufschwung der empirischen Bildungsforschung im deutschsprachigen Raum steht in einem systematischen Zusammenhang mit den als problematisch beurteilten Leistungsergebnissen der Schüler*innen bei den international vergleichenden Leistungsstudien PISA ${ }^{1}$ und teils TIMSS ${ }^{2}$. Auch wenn die Wurzeln der empirischen Bildungsforschung viel weiter zurückliegen (Gräsel, 2011) und auch wenn bereits vor PISA und TIMSS wesentliche Baustellen im Bildungswesen empirisch belegt werden konnten (z.B. in Bezug auf Bildungsbenachteiligungen), haben die ersten PISA- und TIMSS-Ergebnisse in den deutschsprachigen Ländern dennoch grosse Irritation ausgelöst, da damit die Erkenntnis verbunden war, dass das eigene Bildungssystem im internationalen Vergleich nicht zu den besten gehört.

Diese Erkenntnis kann aber nur zum Teil den Aufschwung der empirischen Bildungsforschung im deutschsprachigen Raum erklären. So steht dieser auch in einem Zusammenhang mit neuen Konzepten der Bildungssteuerung bzw. einer Stärkung der Output-Orientierung (bei gleichzeitiger Beibehaltung der bisherigen Steuerungsinstrumente) (Altrichter \& Maag Merki, 2016) und einem verstärkten Qualitätsbewusstsein und ökonomischen Effektivitätskriterien, die ebenfalls seit gut 20 Jahren Eingang in den Bildungsbereich gefunden haben (Terhart, 2000).

Auch wenn bildungspolitische und bildungsadministrative Entscheide bei weitem nicht immer, vielleicht sogar eher selten, auf tatsächlichen empirischen Befunden beruhen - und diese oftmals sogar einzig ex post als Begründung herangezogen werden (Tillmann et al., 2008) - erforderte die verstärkte Evidenzorientierung Erkenntnisse zu den Bedingungsfaktoren, Prozessen und Wirkungen von schulischen Innovationen und Reformen, wodurch empirische Forschung zu diesen Aspekten in breitem Masse gefördert wurde. Und zu guter Letzt, aber nicht weniger bedeutend, gab es in der wissenschaftlichen Community, insbesondere in der Erziehungswissenschaft, der Pädagogischen Psychologie, der Bildungsökonomie und den Fachdidaktiken ein starkes genuines Interesse an einem besseren Verstehen von Bildungsergebnissen im Mehrebenensystem, $d$. h. unter Berücksichtigung des komplexen Zusammenspiels zwischen der bildungspolitischen Ebene, den Einzelschulen und den Unterrichtsprozessen innerhalb der einzelnen Schulen.

Der Aufschwung hätte aber nicht die heute festzustellenden nachhaltigen Wirkungen erreicht, wenn dieser nicht gleichzeitig mit umfangreichen strukturellen Massnahmen und massiven finanziellen Mitteln einhergegangen wäre. Dazu gehörte beispielsweise in Deutschland der Ausbau von Lehrstühlen für empirische Bildungs-

\footnotetext{
1 Programme for International Student Assessment (PISA)

2 Trends in International Mathematics and Science Study (TIMSS)
} 
forschung an vielen Universitäten, die Gründung von international sichtbaren Instituten der Bildungsforschung und Fachdidaktik, so beispielsweise das Institut zur Qualitätsentwicklung im Bildungswesen in Berlin (IQB) (2004), die TUM School of Education (2009) in München, das Leibniz-Institut für Bildungsverläufe in Bamberg (LIfBi) (2014), oder die Gründung der Gesellschaft für Empirische Bildungsforschung (GEBF) (2012).

Diese Entwicklungen können insbesondere in Deutschland und teils in Österreich beobachtet werden, wobei in Österreich vor allem das Bundesinstitut für Bildungsforschung, Innovation \& Entwicklung des österreichischen Schulwesens (BIFIE) als Hinweis auf den strukturellen Ausbau der empirischen Bildungsforschung interpretiert werden kann (dieses heisst seit Juli 2020 neu «IQS - Institut des Bundes für Qualitätssicherung im österreichischen Schulwesen»).

In der Schweiz hingegen zeigen sich nur in Teilen entsprechende Entwicklungen, wobei vor allem der Umfang struktureller Massnahmen oder die Bereitstellung finanzieller Ressourcen deutlich weniger realisiert worden sind. Eine stärkere Evidenzorientierung in der Bildungspolitik und Bildungsadministration, ein Ausbau der empirischen Forschung in der Erziehungswissenschaft, der Aufbau von Doktoratsprogrammen in Fachdidaktik, die Etablierung von (empirischen) Fachdidaktiken an Pädagogischen Hochschulen und Universitäten oder jüngst im Zusammenhang mit den ÜGK - Überprüfung der Grundkompetenzen - die Gründung des Interfaculty Centre for Educational Research (ICER) an der Universität Bern, tragen aber ebenfalls dazu bei, dass die empirische Bildungsforschung in der Schweiz zunehmend sichtbar wurde.

In diesem Artikel soll nun zunächst eine Auseinandersetzung mit der «empirischen Bildungsforschung» als Forschungsfeld realisiert werden, dies aus konzeptioneller, theoretischer und methodischer Perspektive. Die Fokussierung auf die «empirische» Bildungsforschung erfolgt insbesondere darum, weil die empirische Perspektive ein zentrales Element der oben beschriebenen Bildungsforschung ist (Gräsel, 2011), auch wenn zur Bildungsforschung, vor allem, wenn man die Kernfragestellungen nach den «Voraussetzungen, Prozessen und Ergebnissen von Bildung über die Lebensspanne innerhalb und ausserhalb von Institutionen» (Prenzel, 2005) im Blick hat, durchaus auch nicht-empirische Analysen gehören. In einem zweiten Schritt sollen aktuelle Forschungslücken und der Forschungsbedarf identifiziert werden, dies aus theoretischer, methodischer, empirischer und struktureller Perspektive. Dabei wird besonders die empirische Bildungsforschung in der Schweiz (Deutschschweiz) im Fokus stehen.

\section{Empirische Bildungsforschung}

\subsection{Forschungsgegenstand der empirischen Bildungsforschung}

Der Forschungsgegenstand der Bildungsforschung umfasst Fragen zu individuellen Bildungsprozessen, die als Ergebnis des Zusammenspiels individueller Faktoren, sozialer Konstellationen sowie institutioneller Kontexte verstanden werden. So untersucht Bildungsforschung gemäss Baumert (2016)

Bildungsprozesse - einschliesslich ihrer Ziele und Ergebnisse - (...) nicht nur auf individueller Ebene, sondern auch in sozialen Zusammenhängen, die eine Mehrebenenstruktur aufweisen und von der sozialen Nahumwelt wie der Familie und dem Freundeskreis über institutionelle Kontexte bis zu gesamtgesellschaftlichen Zusammenhängen und ihren Veränderungen reichen. In einer diachronen Perspektive schließt empirische Bildungsforschung auch historische Fragestellungen ein. In der Forschungspraxis behandelt die empirische Bildungsforschung in der Regel spezifische Fragen, die im Anschluss an die einschlägige Forschungsliteratur und oftmals auch in Referenz zu politischen oder praktischen Problemlagen theoretisch entwickelt und begründet werden. (Baumert, 2016, S. 217)

Im Zentrum stehen nicht nur Kognitionen, sondern auch motivationale, emotionale oder volitionale Dimensionen des Lernens, wobei oftmals, beispielsweise mit Bezug zu Theorien des selbstregulierten Lernens (z.B. Panadero, 2017), nicht nur die einzelnen Dimensionen separat in den Blick genommen werden, sondern explizit auch das Zusammenspiel mehrerer dieser für das Lernen zentralen Dimensionen untersucht wird. Es interessieren darüber hinaus nicht nur die Ausprägungen dieser Dimensionen, sondern explizit auch deren Veränderungen über die Zeit, um Fragen nach der Stabilität bzw. Veränderung untersuchen zu können.

Hinsichtlich der Analyse der Bedingungsfaktoren individueller Entwicklungen stehen insbesondere individuelle Merkmale im Zentrum, so beispielsweise der sozioökonomische Status, der Migrationshintergrund oder das Geschlecht der Schüler*innen. Diese werden darüber hinaus im Zusammenspiel mit weiteren Faktoren des Elternhauses und insbesondere mit unterrichts- und schulbezogenen Faktoren untersucht. Die Analyse der Lernumgebungen, konzipiert als Mehrebenenstruktur des Bildungswesens, spielt dabei eine entscheidende Rolle. Damit ist das Verständnis gemeint, dass Schüler*innen in Klassen gemeinsame Lernumgebungen im 
Sinne eines differenziellen Lern- und Entwicklungsmilieus erfahren (Baumert et al., 2006), was sich auf die individuellen Lern- und Bildungsprozesse auswirkt. Darüber hinaus zeigt sich, dass die Klassen in einer Schule ein gemeinsames spezifisches schulisches Milieu erleben, so dass in der Folge individuelle Entwicklungen nicht nur durch individuelle Charakteristika und Klassenmerkmale, sondern auch als Ergebnis sozialer Kontexte wie Schulen verstanden werden können (Ditton, 2017; Wurster \& Feldhoff, 2019). Zudem sind auch nationale Bildungssysteme soziale Kontexte, die differenzielle Effekte bewirken und in der Lage sind, Unterschiede in den Leistungen der Schüler*innen zu erklären (Brunner et al., 2018), auch wenn diese als distal bezeichnete Faktoren einen geringeren Erklärungswert für das Verstehen von Unterschieden in den Lernergebnissen der Schüler*innen aufweisen als proximale Faktoren wie der Unterricht oder die Kompetenzen der Lehrpersonen. Somit ist die Mehrebenenlogik mehrschichtig und umfasst nicht nur zwei, sondern drei oder sogar vier Ebenen, was empirisch zu komplexen Analysestrategien führt.

Trotz der grossen Breite möglicher Themenstellungen, die unter Berücksichtigung der Definition von Bildungsforschung denkbar sind, ist in der konkreten Forschungspraxis allerdings eine Fokussierung auf einzelne Themenbereiche zu beobachten. Dies mag zwar vorerst ein subjektiver Eindruck sein, der natürlich einer systematischen Überprüfung bedarf. Allerdings zeigt in einer ersten Annäherung eine Wörter-CloudAnalyse der Beitragsthemen an der (letztlich wegen der Covid-19-Pandemie nicht durchgeführten) Jahrestagung der Gesellschaft für Empirische Bildungsforschung (GEBF) in 2020, dass es durchaus Kernthemen gibt, die im Zentrum der empirischen Bildungsforschung stehen (vgl. Abbildung 1). Wie eingangs erwähnt, kann die GEBF, gegründet im Zuge des Auf- und Ausbaus der empirischen Bildungsforschung im deutschsprachigen Raum, als ein wichtiger Ort im deutschsprachigen Raum betrachtet werden, wo empirische Bildungsforscher*innen ihre Forschungsarbeiten vorstellen und diskutieren.

Die Analyse in Abbildung 1 zeigt eine Schwerpunktsetzung insbesondere hinsichtlich Fragen zur Lehrkraft, zum Unterricht und zum Lernen von Schüler*innen, zu den Kompetenzen und zum Thema Bildung, zur sozialen Dimension des Lernens, zur Digitalisierung sowie zur Ausbildung von Lehrpersonen. Lehr-Lernforschung, Unterrichtsforschung und die Forschung zur Lehrer*innenbildung sind somit corner stones der aktuellen empirischen Bildungsforschung, währenddem beispielsweise Fragen zum Einfluss von Schule als Organisation auf das Lernen der Schüler*innen oder zum Bildungssystem weniger stark in der deutschsprachigen empirischen Bildungsforschung untersucht werden. Darüber hinaus ist die empirische Bildungsforschung stark auf die Schulzeit bis zum Ende der Sekundarstufe II fokussiert, währenddem Bildungsprozesse im weiteren Lebensverlauf weniger untersucht zu sein scheinen. Interessant wäre zu analysieren, ob sich im Zeitverlauf diese Schwerpunktthemen verändert haben. Darüber hinaus kann aus der Themensetzung nicht unmittelbar auf die zu untersuchenden Fragestellungen geschlossen werden, so dass eine Analyse der Fragestellungen noch einen differenzierteren Blick auf das Forschungsfeld der empirischen Bildungsforschung geben könnte.

\section{Abbildung 1 \\ Wörter-Cloud mittels der Titel der Kongressbeiträge am Jahreskongress der GEBF 2020 (https://www.conftool. prolgebf2020/index.php?page =browseSessions Sopath =adminSessions Soprint=exporterismobile =false [Zugriff am 21.12.2020])}

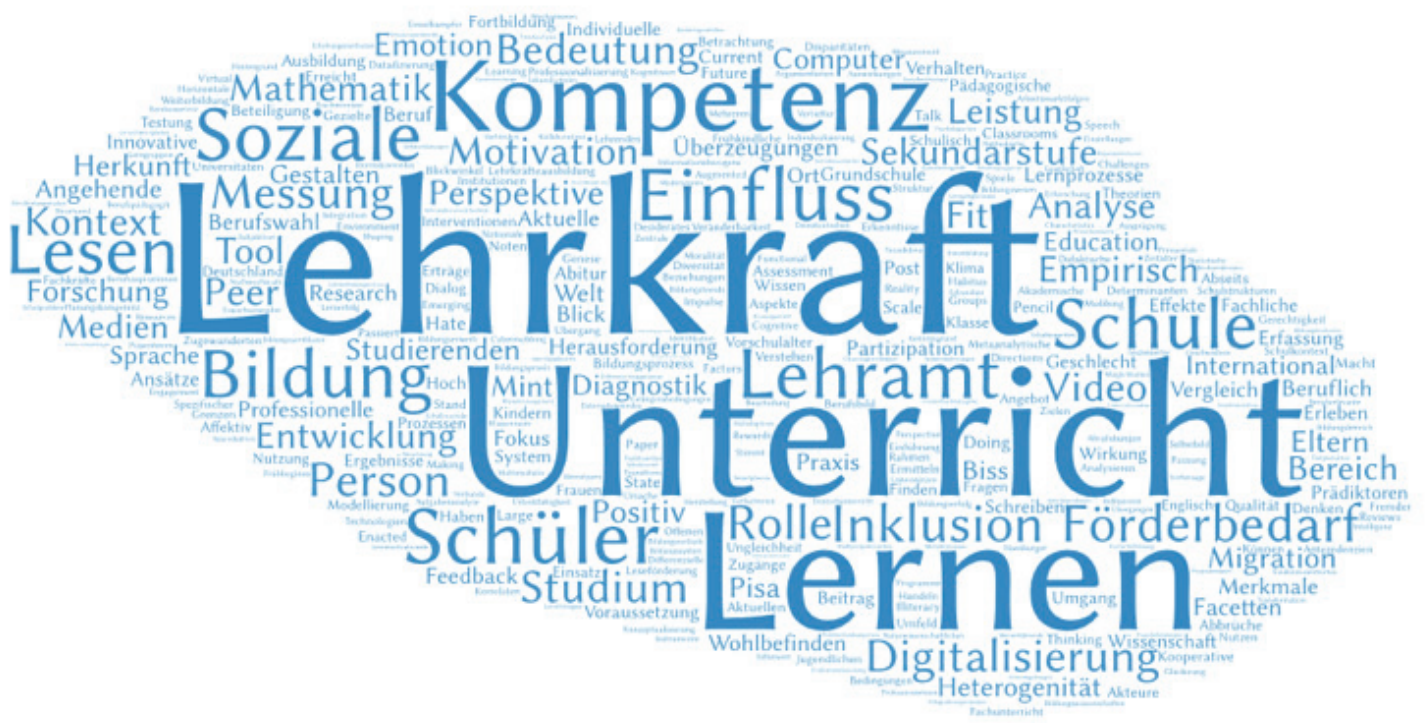

2021 sZBW 43 (1), DOI $10.24452 / \mathrm{sjer} .43 .1 .4$ 


\subsection{Empirische Bildungsforschung als interdisziplinäres Forschungsfeld}

Empirische Bildungsforschung im deutschsprachigen Raum hat sich als Forschungsfeld etabliert, welches aber nicht einer einzelnen Disziplin, beispielsweise der Erziehungswissenschaft, zugeordnet werden kann, sondern genuin interdisziplinär ausgerichtet ist (Gräsel, 2011; Terhart, 2016). Ein Blick in die letzten Jahrzehnte zeigt, dass die Erziehungswissenschaft zwar über lange Zeit leitende Disziplin war, dass aber die Pädagogische Psychologie, genauer die Psychologie des Lehrens und Lernens oder die Diagnostische Psychologie in den letzten Jahren deutlich an Relevanz gewonnen hat, dies im Gegensatz zur Bildungssoziologie, die eher an Gewicht verloren hat (Terhart, 2016). Darüber hinaus ist die Fachdidaktik, insbesondere die empirischen Fachdidaktiken, als eine zentrale Bezugsdisziplin 'aufgestiegen', da das fachliche Lernen und die entsprechende Kompetenzentwicklung eng mit Fragen der Fachdidaktik verknüpft sind. Darüber hinaus können auch Bildungsökonomie und Politikwissenschaft als Bezugsdisziplinen der empirischen Bildungsforschung verstanden werden, insbesondere dann, wenn Fragen zu den Effekten der Bildungssteuerung auf das Lehren und Lernen untersucht werden. Allerdings ist das Gewicht der Bildungsökonomie und der Politikwissenschaft nicht vergleichbar mit jenem der Erziehungswissenschaft, Psychologie und der Fachdidaktiken. Zudem kann vermutet werden, dass die Identifikation der Forscher*innen der Bildungsökonomie und der Politikwissenschaft mit der empirischen Bildungsforschung weniger stark ausgeprägt ist als jene der Erziehungswissenschaft, Psychologie oder der Fachdidaktiken, was sich auch in einer geringeren Präsenz an den Jahreskongressen der GEBF zeigt.

\subsection{Empirische Bildungsforschung als methodischer Ansatz}

Aus einer methodischen Perspektive wird die empirische Bildungsforschung eng mit standardisierten quantitativen Forschungsmethoden, insbesondere mit den in den letzten 20 Jahren durchgeführten Large-ScaleAssessment-Studies (LSA) assoziiert. Zu diesen gehören auf internationaler Ebene beispielsweise PISA oder PIRLS 3 . Aber auch auf nationaler Ebene (z.B. in Deutschland das Nationale Bildungspanel (NEPS), Civic Education oder Teacher Education and Development Study in Mathematics (TEDS-M)) bilden diese Studien die Grundlage für die Bearbeitung einer grossen Anzahl an Forschungsfragen, die der empirischen Bildungsforschung zugeordnet werden können (Baumert, 2016). Für die Schweiz interessant sind insbesondere die Studie «Transitionen von der Erstausbildung ins Erwerbsleben» (TREE) oder seit neustem die Erhebungen im Zusammenhang mit der «Überprüfung der Grundkompetenzen» (ÜGK).

Nichtsdestotrotz ist empirische Bildungsforschung nicht primär auf ein standardisiertes methodisches Vorgehen eingegrenzt, enthält somit gemäss Baumert (2016, S. 217) keine methodischen Präferenzen. Damit ist sie grundsätzlich auch offen für qualitative Forschung oder historische Fragestellungen. Allerdings ist kaum zu übersehen, dass in der aktuellen Forschung eine Orientierung an dem standardisierten, hypothesenüberprüfenden Forschungsparadigma dennoch deutlich überwiegt, sichtbar beispielsweise wiederum an den Kongressbeiträgen der Jahreskongresse der Gesellschaft für Empirische Bildungsforschung (GEBF) in den letzten Jahren.

Damit ergibt sich eine Diskrepanz zwischen Forschungsprogramm und konkreter Forschungspraxis. Dies kann vielfältige Gründe haben. Einer der Gründe neben anderen (Tillmann, 2016) dürfte sein, dass Forscher*innen, deren Arbeiten zwar anschlussfähig an die oben formulierten Forschungsfragen der empirischen Bildungsforschung (als Forschungsfeld) wären, aber ein qualitatives Forschungsparadigma verfolgen, sich nicht als «empirische Bildungsforscher*innen» identifizieren und in der Folge beispielsweise die Tagungen der GEBF weniger besuchen oder eigene Tagungen organisieren, was zu getrennten Diskursen und wissenschaftlichen Gemeinschaften führt. Wie Helsper (2016) zudem am Beispiel der Bildungsungleichheitsforschung aufzeigen konnte, bleibt zudem fraglich, inwiefern sich die als eher getrennt agierenden communities der quantitativen und qualitativen Bildungsforscher*innen dennoch gegenseitig wahrnehmen und die unterschiedlichen methodischen Ansätze und teils auch Ergebnisse im Sinne der Triangulation nutzen, um die Forschungsfragen integral zu erörtern. Zugespitzt würde das heissen, dass «empirische Bildungsforschung» zwar theoretisch ein Forschungsfeld ist, in der Praxis aber als ein spezifisches, nämlich standardisiertes Forschungsparadigma wahrgenommen wird. Dies kann kaum eine gute Grundlage für den Erkenntnisgewinn bei den zu untersuchenden Forschungsfragen der Bildungsforschung sein, gerade auch wenn berücksichtigt wird, dass alle methodischen Vorgehen je ihre eigenen Stärken und Schwächen aufweisen und ein interdisziplinärer Ansatz nicht nur inhaltlich und theoretisch, sondern auch methodisch gewinnbringend wäre.

3 Progress in International Reading Literacy Study (PIRLS) 


\subsection{Ziele der empirischen Bildungsforschung}

Neben dem empirischen Zugang und der Interdisziplinarität als zentrale Merkmale empirischer Bildungsforschung kann die Problemorientierung als weiteres bedeutsames Merkmal bezeichnet werden. Dabei werden nicht nur die Problemlagen der Praxis, sondern auch jene der Bildungspolitik in den Blick genommen, wobei die zentrale Aufgabe der Bildungsforschung darin gesehen wird, "wissenschaftliche Erkenntnisse zu gewinnen, die zu einer Analyse und der Verbesserung des Bildungswesens beitragen» (Gräsel, 2011, S. 14). Diese Politiknähe wird zuweilen auch von Akteur*innen insbesondere aus der Erziehungswissenschaft massiv kritisiert, wie dies das Sonderheft der Zeitschrift für Erziehungswissenschaft dokumentiert (Baumert \& Tillmann, 2016). So stellt sich tatsächlich die Frage, inwiefern empirische Forschung zur Verbesserung der Bildungspraxis (auf unterschiedlichen Ebenen des Bildungssystems) beitragen kann.

Zunächst ist festzuhalten, dass der Anspruch an die Bildungsforschung, einen Beitrag zur Verbesserung des Bildungssystems und der Bildungspraxis zu leisten, in hohem Masse gerechtfertigt ist. Allerdings sind die Ziele von Forschung vielfältiger und die Möglichkeiten, belastbare empirische Befunde in einen unmittelbaren Zusammenhang mit möglichen Schritten der Qualitätsentwicklung des Bildungswesens zu stellen, eingeschränkt.

Empirische Bildungsforschung dient zum einen der Beschreibung von Bildungsprozessen und Bildungsergebnissen. So zeigen beispielsweise gerade die umfangreichen Befunde im Zusammenhang mit den standardisierten (inter)national vergleichenden Studien, dass sich die Qualität der Lernumgebungen, sei dies auf Klassen-, Schul-, Regionen- oder Nationenebene, deutlich voneinander unterscheidet (Brunner et al., 2018; Ditton, 2017; Kyriakides \& Creemers, 2009; Reynolds et al., 2014; Wurster \& Feldhoff, 2019), dass gerade im deutschsprachigen Bildungsraum ein deutlicher Zusammenhang von Bildungserfolg mit dem familiären sozio-kulturellen Hintergrund der Schüler*innen besteht (Kriesi \& Leemann, 2020) oder dass in der Schweiz die theoretisch umfangreichen Möglichkeiten der Durchlässigkeit nach Ende der obligatorischen Schulzeit in der Praxis nicht oder nur wenig genutzt werden (vgl. z. B. Scharenberg et al., 2014; Scharenberg et al., 2017). Das Sichtbarmachen dieser Spannungsfelder und Herausforderungen ist wesentlich und kann als erster, absolut zentraler Schritt für eine Verbesserung der Bildungspraxis betrachtet werden, zumal mit den Ergebnissen der Analysen beispielsweise deutlich wird, dass sich Fragen zur Chancenungleichheit im Bildungswesen weiterhin akzentuieren, was gerade auch für die Schweiz diskrepant zum von der Erziehungsdirektorenkonferenz (EDK) formulierten Ziel des Bildungswesens steht (siehe https:/www.edk.ch/dyn/14798.php; [Zugriff am 21.12.2020]).

Zum anderen ist ein wesentliches Ziel von Bildungsforschung, Erklärungswissen und Wissen zu Bedingungen von Entwicklungs- und Veränderungsprozessen zu generieren. Die Fragen nach der Erklärung, nach Ursache-Wirkungszusammenhängen und nach dem Verändern möglicher Spannungsfelder und Problemzonen sind allerdings ungleich schwieriger zu beantworten als die Beschreibung dieser Spannungsfelder und Problemzonen (vgl. beispielsweise bezüglich der Effekte der Einführung zentraler Abiturprüfungen: Maag Merki, 2014). Gerade Large-Scale-Assessment-Studies sind oftmals nicht in der Lage, genügend Erklärungswissen für allfällige Unterschiede zwischen Schüler*innen, Klassen, Regionen oder Nationen zu generieren. Hierzu sind vertiefende Analysen, gerade auch mit qualitativen Forschungsmethoden, Interventionsstudien oder mixed-methodsDesigns notwendig.

Sollten sich Ursachen für nicht zufriedenstellende Effekte identifizieren lassen, so zeigt die internationale Literatur der Schulentwicklungsforschung wiederum, dass es, trotz der in den letzten Jahren gewonnenen Erkenntnissen zu zentralen Gelingensfaktoren von Schulentwicklung (Maag Merki, im Druck), weder Rezepte für Qualitätsentwicklung noch Strategien gibt, die sich situationsübergreifend durchgehend bewähren. Situationsspezifität, Adaptivität und Komplexität sind Stichworte, die darauf verweisen, dass die systematische Verbesserung von Lernumgebungen mit dem Ziel der Erreichung besserer Bildungsergebnisse ein anspruchsvoller, mehrjähriger Prozess ist, der zudem abhängig ist von strukturellen Rahmenbedingungen und dem kompetenten Handeln vielfältiger Akteure - Voraussetzungen, die in den seltensten Fällen gegeben sind. Darüber hinaus passen die Zeitperspektiven bildungspolitischer Erwartungen und bildungswissenschaftlicher Analysen selten zusammen, da bildungspolitische Akteure oftmals in kurzer Zeit (positive) Effekte ihrer Entscheide empirisch 'bewiesen' haben möchten, während die Analyse der Nachhaltigkeit von Effekten wissenschaftlich ein Langzeitunternehmen ist. In dem Sinne können durchaus Situationen entstehen, wo der Schnittbereich von Bildungsforschung und Bildungspolitik relativ gering ist, oder wo Bildungsforschung für bildungspolitische Entscheide instrumentalisiert wird oder sich instrumentalisieren lässt. 


\section{Perspektiven zukünftiger Entwicklungen der empirischen Bildungsforschung mit einem spezifischen Fokus auf die Schweiz}

Ausgehend von den Erfahrungen in den letzten Jahren lassen sich mehrere Desiderata identifizieren, die leitend für die zukünftigen Entwicklungen der empirischen Bildungsforschung sein könnten. Hierzu gehören theoretische-konzeptionelle Klärungen, methodische Herausforderungen, Forschungsdesiderata bezüglich der Klärung inhaltlicher Fragestellungen und die Frage nach strukturellen Anforderungen an die zukünftige empirische Bildungsforschung, insbesondere in der Schweiz.

\subsection{Herausforderungen bezüglich theoretischer und konzeptioneller KLärungen}

Inwiefern Bildungsforschung als Forschungsfeld tatsächlich zum Erkenntnisgewinn bezüglich des Verstehens und Erklärens von Bildungsprozessen beitragen kann, hängt langfristig damit zusammen, wie dieses Forschungsfeld theoretisch belastbar beschrieben und konzipiert werden kann, wie also die verschiedenen Teilgebiete der Bildungsforschung nicht nur in sich, sondern auch mit Bezug zueinander, theoretisch fundiert werden können. Dies scheint bislang noch nicht gelungen zu sein. Es zeigen sich zwar in den je spezifischen Forschungsfeldern, beispielsweise in der Unterrichtsforschung (z. B. Praetorius et al., 2020) oder der Lehrer*innenbildungsforschung (z. B. Cramer et al., 2020), je eigene Theoriemodellierungen, aber eine integrale Theorie der Bildungsforschung besteht nicht. $\mathrm{Ob}$ es diese je geben wird, ist unklar. $\mathrm{Ob}$ es überhaupt angesichts der Komplexität des Gegenstandes möglich ist, eine solche zu entwickeln, ist ebenfalls unklar. Und es stellt sich auch die Frage, inwiefern der Anspruch, eine solche Theorie zu entwickeln, als Ziel verfolgt werden kann und soll. Denn, etwas provokativ formuliert: Braucht es eine Theorie oder ein Konzept «empirischer Bildungsforschung», wenn es «empirische Unterrichtsforschung», «empirische Lehr-Lernforschung» oder «empirische Schulforschung» gibt? Bildungsforschung kann als Dach empirischer Forschung im Bildungsbereich generell figurieren. Dieser Zusammenschluss empirischer Forschung ist für sich ertragreich, so beispielsweise, wenn Bildungsprozesse unter unterschiedlichen disziplinären Fragestellungen untersucht werden und wenn ein Ort der Diskussion entstehen kann, an dem, nebeneinander oder miteinander, die vielfältigen Fragen erörtert werden. Aber mit Rückblick auf bisherige Forschung könnte auch der Eindruck der Beliebigkeit entstehen. Falls es nicht gelingt, eine Konturierung des Forschungsfeldes und der Forschungsfragen vorzunehmen, bleibt der Eindruck eines «Sammeltopfes» zurück und die Frage akzentuiert sich, welche Forschungsfragen denn tatsächlich Forschungsfragen der (empirischen) Bildungsforschung sind (und nicht einzig der (empirischen) Unterrichtsforschung oder Schulforschung), welches add-on ein Konzept empirischer Bildungsforschung gegenüber Unterrichtsforschung oder Schulforschung aufweist und wer von den Bildungsforscher*innen, die einen empirischen Forschungsansatz verfolgen, denn eigentlich NICHT zur empirischen Bildungsforschung gehört.

\subsection{Methodische Herausforderungen}

Neben der schon oftmals formulierten Anforderung, stärker mittels unterschiedlicher methodischer Verfahren die Fragen nach den Bedingungen und Effekten von Bildungsprozessen im Längsschnitt zu untersuchen (methodische Pluralität, mixed-methods, Triangulation), stellen sich insbesondere auch Fragen, wie Ursache- und Wirkungszusammenhänge oder komplexe Dynamiken im Dreieck von Handlung, Struktur und Akteurskonstellationen im Mehrebenensystem erfasst werden können und wie beispielsweise schulische Prozesse stärker performanznah untersucht werden können, so dass die Erkenntnisse zum Verstehen von Bildungsprozessen nicht hauptsächlich auf Selbstberichten der befragten Akteure basieren. Time sampling oder experience sampling Methoden, soziale Netzwerkanalysen oder die methodischen Verfahren, die sich aufgrund der neu vorhandenen big data entwickelt haben (z.B. Educational Data Mining, Learning Analytics) sind interessante methodische Weiterentwicklungen, die auch für die empirische Bildungsforschung zunehmend auf ihre Belastbarkeit und ihren Ertrag bezüglich Erkenntnisgewinn geprüft werden. Relevant dürfte zudem sein, noch vertiefter Veränderungen über die Zeit nicht nur mittels einer linearen Logik, sondern auch unter Berücksichtigung nicht-linearer Zusammenhänge und der Qualitäten der Handlungen und Prozesse (und nicht nur der Häufigkeiten) zu untersuchen, wie dies beispielsweise das Dynamic Model of Educational Effectiveness von Creemers and Kyriakides (2008) schon lange postuliert, aber bislang noch zu wenig in der konkreten Forschungspraxis umgesetzt ist (auch von Creemers und Kyriakides nicht). Darüber hinaus bleibt die Realisierung von ökologisch validen Interventionsstudien als eine der zentralen Herausforderungen empirischer Bildungsforschung, um noch stärker Ursache-Wirkungszusammenhänge sowie komplexere Zusammenhänge über die Zeit erschliessen zu können. 


\subsection{Inhaltlich-fachliche Forschungsdesiderata}

An dieser Stelle wird es nicht möglich sein, auf einzelne fachliche Forschungsdesiderata eingehen zu können. Wird die theoretische Konzipierung von Bildungsforschung (vgl. z. B. Baumert, 2016, S. 217) aber ernst genommen, so lassen sich zum einen wesentliche Forschungsdesiderata besonders hinsichtlich des Zusammenspiels sozialer Zusammenhänge im Mehrebenensystem identifizieren. So könnte verstärkt interessieren, wie dieses Zusammenspiel theoretisch konzipiert, empirisch erfasst und inhaltlich ausgestaltet ist. Zum anderen stellt sich dann natürlich die Frage, welche Effekte dieses Zusammenspiel auf die Entwicklungs- und Bildungsprozesse der Individuen haben und welche Konfigurationen gelingende oder problematische Entwicklungen zur Folge haben. Die kleine Analyse der Themengebiete, die im Rahmen der Jahrestagung der GEBF in 2020 als Forschungsbeiträge berücksichtigt wurden, könnte zudem als Anlass genommen werden zu prüfen, welche Fragebereiche fokussiert und welche eher peripher bearbeitet werden und inwiefern die unterschiedliche Gewichtung von Forschungsfragen einen Einfluss auf das Verständnis von Bildungsprozessen haben könnte.

\subsection{Aufbau einer Forschungsinfrastruktur für empirische Bildungsforschung}

Die Realisierung von empirischer Bildungsforschung benötigt entsprechende Rahmenbedingungen. Eine der wesentlichsten Rahmenbedingungen ist die Möglichkeit, Bildungsprozesse vor Ort, in den Klassen, Schulen, an ausserschulischen Orten, in Familien, Institutionen etc. zu untersuchen. Dabei haben sich in den letzten Jahren deutliche Probleme ergeben. Eines der grössten Probleme zeigt sich in Bezug auf das Gewinnen von Lehrpersonen, Schulleitungen, Schüler*innen, Jugendlichen für eine Teilnahme an den geplanten Studien. Es ist offensichtlich: Ohne belastbare Stichproben können keine Forschungsfragen angemessen beantwortet werden. Es ist aber auch offensichtlich, dass die Akteure nur begrenzt Zeit einsetzen können, sich an Forschungsstudien zu beteiligen, sogar auch dann, wenn sie grundsätzlich an den Forschungsfragen interessiert sind und offen sind gegenüber empirischer Forschung. Diese Problemlage hat sich in den letzten Jahren noch akzentuiert, so dass es teilweise kaum mehr möglich ist, auch mit qualitativ exzellenten Forschungsdesigns und Erhebungsinstrumenten, beispielsweise schulische Akteure für eine Teilnahme zu gewinnen.

Damit hängt auch ein zweites Problem zusammen, nämlich das Problem, dass, zumindest im schulischen Feld, viele Kleinststudien mit mangelnder Qualität durchgeführt werden, die nur einen begrenzten Erkenntnisgewinn erlauben. Für die Zukunft der empirischen Forschung im Bildungsbereich ist es essentiell, Lösungen für diese Probleme zu finden.

Eine Möglichkeit wäre, vermehrt aufbereits bestehende Datensätze zurückzugreifen. Entwicklungen bezüglich der Bereitstellung von bestehenden Daten für die Sekundäranalyse bestehen bereits seit langem (für die Schweiz, https://forsbase.unil.ch/; Zugriff am 21.12.2020). Allerdings ist damit allenfalls die Schwierigkeit verknüpft, dass neue Forschungsfragen nicht wirklich bearbeitet werden können, da alte / ältere Konstrukte verwendet wurden und aus diesem Grund auch nur bereits bestehende Forschungsfragen aus anderer Perspektive reanalysiert werden können. Dennoch ist das ein sehr zentrales Standbein der zukünftigen empirischen Bildungsforschung, die noch ausgebaut werden kann, zumal in den grossen Datensätzen, die beispielsweise bei FORSBASE verfügbar sind, kaum alle Fragestellungen bereits untersucht worden sind.

Besonders wichtig erscheint auch eine stärkere Koordination der Forschungsprojekte, die in Bezug auf eine gemeinsame Thematik geplant und durchgeführt werden. Gerade im Zusammenhang mit der Analyse von Forschungsfragen bezüglich der Covid-19-Pandemie hat sich diese Notwendigkeit einer Koordination in hohem Masse akzentuiert. Eine entsprechende online-Plattform hätte die Chance, einen Austausch zwischen den Forscher*innen zu fördern, zumal ansonsten häufig erst im Nachhinein Informationen zu entsprechenden Forschungsprojekten bereitgestellt sind. Aus diesem Grund ist der Aufbau einer entsprechenden Plattform an der ETH Lausanne sehr zu begrüssen (https://airtable.com/shrQFS0CG3jdPf725/tblbgmyj6f8HAiKYo; Zugriff am 21.12.2020). Besonders positiv ist, dass der Aufbau dieser Forschungsinfrastruktur durch die Erziehungsdirektorenkonferenz (EDK), das Staatssekretariat für Bildung, Forschung und Innovation (SBFI) und die ETH Lausanne gemeinsam realisiert wurde. Dies ist ein wichtiger Pfeiler für die nachhaltige Realisierung von empirischer Bildungsforschung in der Zukunft.

Für eine zukunftsstarke Bildungsforschung könnte aber auch darüber nachgedacht werden, bei der Konzipierung von Studien a-priori unterschiedliche Fragestellungen von unterschiedlichen Forscher*innen zu integrieren, so dass nicht jede der Forscher*innen separat entsprechende Schulen für eine Teilnahme gewinnen müssten, sondern gemeinsam eine belastbare Stichprobe gewonnen werden kann. In der Schweiz wurde dies bereits im Rahmen der TREE-Studie realisiert; für den vorschulischen oder schulischen Bildungsbereich scheint dies aber noch zu fehlen. Forschungsverbünde könnten hier eine interessante Basis sein, damit diese Forschungsprojekte im Forschungsfeld breit verankert sind und allfällige Konkurrenzsituationen angemessen bearbeitet 
werden können. Eine Möglichkeit wäre darüber hinaus, dass das ICER, das Interfaculty Centre for Educational Research an der Universität Bern, das im Zusammenhang mit der Überprüfung der Grundkompetenzen entstanden ist, eine führende Rolle übernehmen würde. Sicherlich, dieser Ansatz ist anspruchsvoll, aber eine stärkere Koordination der Erhebungen könnte einen wesentlichen Gewinn darstellen.

Für exzellente Bildungsforschung zeigen sich zudem die Möglichkeiten des Bundesamtes für Statistik als besonders relevant, insbesondere dann, wenn individuelle Entwicklungsverläufe nachgezeichnet und untersucht werden sollen. In Bezug auf Fragen, die die Lernkontexte betreffen, beispielsweise schulische Institutionen, reichen die bestehenden Indikatoren allerdings noch nicht aus, um längsschnittliche Analysen auch auf Schul- oder generell Institutionenebene und der Einfluss der Schulebene auf die Entwicklungsverläufe zu analysieren. Hier wäre wünschenswert, wenn die Möglichkeiten der standardisierten Datengewinnung noch verfeinert und ausgebaut würden.

\section{Literatur}

Altrichter, H. \& Maag Merki, K. (2016). Steuerung der Entwicklung des Schulwesens. In H. Altrichter \& K. Maag Merki (Hrsg.), Handbuch Neue Steuerung im Schulsystem, 2., erweiterte Ausgabe (S. 1-28). Springer VS.

Baumert, J. (2016). Leistungsgrenzen der empirischen Bildungsforschung. Das Beispiel von Large-Scale-Assessment-Studien zwischen Wissenschaft und Politik. Zeitschrift für Erziehungswissenschaft, 19 (Suppl I), 215-253. https://doi.org/10.1007/ s11618-016-0704-4

Baumert, J., Stanat, P. \& Watermann, R. (2006). Schulstruktur und die Entstehung differenzieller Lern- und Entwicklungsmilieus. In J. Baumert, P. Stanat \& R. Watermann (Hrsg.), Herkunftsbedingte Disparitäten im Bildungswesen: Differenzielle Bildungsprozesse und Probleme der Verteilungsgerechtigkeit. Vertiefende Analysen im Rahmen von PISA 2000 (S. 95-188). Springer VS.

Baumert, J. \& Tillmann, K.-J. (Hrsg.). (2016). Empirische Bildungsforschung. Der kritische Blick und die Antwort auf die Kritiker. Zeitschrift für Erziehungswissenschaft, Sonderheft 31. Springer VS.

Brunner, M., Keller, A., Wenger, M., Fischbach, A. \& Lüdtke, O. (2018). Between-school variation in students' achievement, motivation, affect, and learning strategies: results from 81 countries for planning group-randomized trials in education. Journal of Research on Educational Effectiveness, 11(3), 452-478. https://doi.org/10.1080/19345747.2017.1375584

Cramer, C., König, J., Rothland, M. \& Blömeke, S. (Hrsg.). (2020). Handbuch Lehrerinnen- und Lehrerbildung. Julius Klinkhardt.

Creemers, B. P. M. \& Kyriakides, L. (2008). The dynamics of educational effectiveness. A contribution to policy, practice and theory in contemporary schools. Routledge.

Ditton, H. (2017). Zum Wirkungszusammenhang der schulischen Handlungsebenen. In U. Steffens, K. Maag Merki \& H. Fend (Hrsg.), Schulgestaltung. Aktuelle Befunde und Perspektiven der Schulqualitäts- und Schulentwicklungsforschung. Grundlagen der Qualität von Schule 2 (S. 59-84). Waxmann.

Gräsel, C. (2011). Was ist Empirische Bildungsforschung? In H. Reinders, H. Ditton, C. Gräsel \& B. Gniewosz (Hrsg.), Empirische Bildungsforschung. Strukturen und Methoden. Lehrbuch (S. 13-27). VS Verlag für Sozialwissenschaften.

Helsper, W. (2016). Wird die Pluralität in der Erziehungswissenschaft aufgekündigt? Zeitschrift für Erziehungswissenschaft, 19 (Suppl I), 89-105. https://doi.org/10.1007/s11618-016-0709-z

Kriesi, I. \& Leemann, R. J. (2020). Tertiarisierungsdruck. Herausforderungen für das Bildungssystem, den Arbeitsmarkt und das Individuum (Vol. 15).

Kyriakides, L. \& Creemers, B. P. M. (2009). The effects of teacher factors on different outcomes: Two studies testing the validity of the dynamic model. Effective Education, 1(1), 61-85. https://doi.org/10.1080/19415530903043680

Maag Merki, K. (2014). Das quasi-experimentelle Design in der Educational Governance-Forschung? Herausforderungen, Möglichkeiten und Grenzen am Beispiel der Analyse der Wirksamkeit der Einführung zentraler Abiturprüfungen. In K. Maag Merki, R. Langer \& H. Altrichter (Hrsg.), Educational Governance als Forschungsperspektive. Strategien, Methoden, Ansätze; 2. Auflage (S. 51-83). Springer VS.

Maag Merki, K. (im Druck). Schulentwicklungsforschung. In T. Hascher, T.-S. Idel \& W. Helsper (Hrsg.), Handbuch Schulforschung. Springer VS.

Panadero, E. (2017). A review of self-regulated learning: Six models and four directions for research. Frontiers in Psychology, 8(422). https://doi.org/10.3389/fpsyg.2017.00422

Praetorius, A.-K., Grünkorn, J. \& Klieme, E. (2020). Empirische Forschung zu Unterrichtsqualität. Theoretische Grundfragen und quantitative Modellierungen. Zeitschrift für Pädagogik. Beiheft, 9-14. https://doi.org/10.3262/ZPB2001009

Prenzel, M. (2005). Zur Situation der Empirischen Bildungsforschung. In H. Mandl \& B. Kopp (Hrsg.), Impulse für die Bildungsforschung. Stand und Perspektiven. Dokumentation eines Expertengesprächs der DFG (S. 7-22). Akademie Verlag.

Reynolds, D., Sammons, P., De Fraine, B., Van Damme, J., Townsend, T., Teddlie, C. \& Stringfield, S. (2014). School effectiveness research (EER): a state-of-the-art review. School Effectiveness and School Improvement, 25(2), 197-230. https://doi. org/10.1080/09243453.2014.885450

Scharenberg, K., Rudin, M., Müller, B., Meyer, T. \& Hubka-Brunner, S. (2014). Ausbildungsverläufe von der obligatorischen Schule ins junge Erwachsenenalter: Die ersten zehn Jahre. Ergebnisübersicht der Schweizer Längsschnittstudie TREE, Teil I. TREE.

Scharenberg, K., Wohlgemuth, K. \& Hupka-Brunner, S. (2017). Does the structural organisation of lower-secondary education in Switzerland influence students' opportunities of transition to upper-secondary education? A multilevel analysis. Swiss Journal of Sociology, 43(1), 63-87. https://doi.org/10.1515/sjs-2017-0004

Terhart, E. (2000). Qualität und Qualitätssicherung im Schulsystem. Hintergründe - Konzepte - Probleme. Zeitschrift für Pädagogik, 46(6), 809-830. 
Terhart, E. (2016). Empirische Bildungsforschung und ihre Disziplinen - Wandlungsprozesse und Konfliktlinien in instabilen Expertenkulturen. Zeitschrift für Erziehungswissenschaft, 19 (Suppl I), 73-87. https://doi.org/10.1007/s11618-016-0708-0

Tillmann, K.-J. (2016). Empirische Bildungsforschung in der Kritik - ein Überblick über Themen und Kontroversen. Zeitschrift für Erziehungswissenschaft, 19 (Suppl I), 5-22. https://doi.org/10.1007/s11618-016-0705-3

Tillmann, K.-J., Dedering, K., Kneuper, D., Kuhlmann, C. \& Nessel, I. (2008). PISA als bildungspolitisches Ereignis. Fallstudien in vier Bundesländern. Verlag für Sozialwissenschaften.

Wurster, S. \& Feldhoff, T. (2019). Schul- und Unterrichtsqualität aus der Mehrebenenperspektive. Ist die Schule oder die Klasse die relevante pädagogische Gestaltungseinheit? Zeitschrift für Pädagogik, 65(1), 24-39. https://doi.org/10.3262/ZP1901024

Schlagworte: Empirische Bildungsforschung; Mehrebenensystem; historische Entwicklung; Forschungsbedarf; Forschungsinfrastruktur

\section{Recherche empirique en éducation dans l'espace germanophone. Bilan et perspectives}

Résumé

L'expansion de la recherche empirique en éducation dans l'espace germanophone au cours des vingt dernières années est lié aux résultats d'études comparatives internationales sur les acquis scolaires telles que PISA et TIMSS, à la mise en œuvre de nouvelles formes de gouvernance et à des mesures structurelles de grande envergure. Cet article commence par un examen de la «recherche empirique en éducation» en tant que domaine de recherche. Dans un deuxième temps, les lacunes actuelles de la recherche sont décrites. Un besoin de recherche peut être identifié d'un point de vue théorique et méthodologique et en ce qui concerne la clarification des questions sur les facteurs de conditionnement et les effets des processus éducatifs. En outre, le développement d'une infrastructure de recherche pour la recherche en éducation est préconisé.

Mots-clés: Recherche empirique en éducation; système à plusieurs niveaux ; développement historique ; besoins en matière de recherche; infrastructure de recherche

\section{La ricerca educativa empirica nel mondo di lingua tedesca. Stato dell'arte e prospettive}

\section{Riassunto}

La ripresa della ricerca empirica sull'educazione nel mondo germanofono negli ultimi vent'anni è legata ai risultati di studi comparativi internazionali come PISA e TIMSS, all'implementazione di nuove forme di governance e ad ampie misure strutturali. Questo articolo inizia con un esame della «ricerca empirica educativa» come campo di indagine. In una seconda fase vengono descritte le attuali lacune nell'ambito della ricerca empirica educativa. La necessità di ulteriori studi emerge sia sul piano teorico che su quello metodologico in relazione ai fattori che influenzano i processi educativi e agli effetti di tali processi. Si sostiene, inoltre, la necessità di sviluppo di un'infrastruttura per la ricerca educativa.

Parole chiave: Ricerca empirica educativa; sistema multilivello; sviluppo storico; esigenze di ricerca; infrastrutture di ricerca 


\section{Empirical Educational Research in German-speaking Countries. Review and Perspectives}

Summary

The growth of empirical educational research in the German-speaking world over the past twenty years is related to the results of international large-scale assessments such as PISA and TIMSS, the implementation of new forms of governance, and the provision of extensive structural measures. This article first discusses "empirical educational research" as a field of research. In a second step, current research gaps are described. A need for research can be identified from a theoretical and methodological perspective and with regard to clarifying questions about the influencing factors and effects of educational processes. Furthermore, the development of a research infrastructure for educational research is advocated.

Keywords: Empirical education research; multilevel system; historical development; research needs; research infrastructure

Prof. Dr. Katharina Maag Merki, ordentliche Professorin für Theorie und Empirie schulischer Bildungsprozesse an der Universität Zürich; Forschungsschwerpunkte sind Schulentwicklung, Schuleffektivität, Educational Governance, Selbstreguliertes Lernen.

Aktuelle Forschungsprojekte: www.ife.uzh.ch/sic; www.s-clever.org

Universität Zürich, Institut für Erziehungswissenschaft, Freiestrasse 36, CH-8032 Zürich.

E-Mail: kmaag@ife.uzh.ch 